\title{
(C) OPEN ACCESS \\ HIV-associated benign lymphoepithelial cysts of the parotid glands confirmed by HIV-1 p24 antigen immunostaining
}

\author{
Yoshiyuki Sekikawa, Igen Hongo
}

Infectious Diseases, Musashino Red Cross Hospital, Musashino, Tokyo, Japan

Correspondence to Dr Yoshiyuki Sekikawa, yoshiyuki1021@gmail.com

Accepted 19 September 2017

\section{SUMMARY}

Approximately $1 \%-10 \%$ of patients with HIV infection have been reported to have salivary gland enlargement. Parotid swelling in patients with HIV is often associated with salivary gland disease, including benign lymphoepithelial cysts (BLECs). The presence of BLEC can serve as an indicator of HIV infection, and the diagnosis of HIV-associated BLEC is usually based on clinical course, HIV confirmatory blood testing, such as western blot or viral detection, and imaging studies, but not on biopsies or immunostaining. To exclude other diseases such as tuberculosis and malignant lymphoma and to further improve the diagnostic accuracy of BLEC, the detection of the HIV1 p24 antigen by immunohistochemistry is a useful diagnostic method. We report a case of a 65 -year-old Japanese man with swelling of the parotid glands and HIV-associated BLEC confirmed via HIV-1 p24 immunohistochemical staining.

\section{BACKGROUND}

According to European and North American studies, approximately $1 \%-10 \%$ of patients with HIV have salivary gland enlargement. ${ }^{1}$ Parotid swelling in patients with HIV is often associated with salivary gland disease, such as inflammatory disorders, infections, neoplasms and benign lymphoepithelial cysts (BLECs). BLEC is characterised by bilateral parotid gland swelling and cervical lymphadenopathy, and the presence of BLECs can serve as an initial clinical manifestation of HIV.

The first case of BLEC was reported by Hildebrandt in 1895, and until 1981, the period known as the pre-HIV era, only 21 cases had been described. ${ }^{2}$ While the incidence of BLEC has increased with the emergence of HIV, the prevalence of BLEC has decreased with the development of antiretroviral therapy (ART). The diagnosis of HIV-associated BLEC is predominantly made through blood and imaging tests in patients with asymptomatic parotid gland swelling, and to the best of our knowledge only a few reports have shown direct diagnosis of HIV-associated BLEC through immunohistochemical detection of the HIV-1 p24 antigen. In this report, we present a case of a 65 -year-old man positive for HIV infection, who was diagnosed with HIV-associated BLEC through pathological examination and immunohistochemical detection of the HIV-1 p24 antigen.

\section{CASE PRESENTATION}

A 65-year-old Japanese man presented with a 12-month history of bilateral, painless parotid gland swelling. Contrast-enhanced CT showed cervical, supraclavicular, mediastinal and hilar lymphadenopathy, in addition to bilateral parotid gland swelling. HIV screening tests indicated that the patient was positive for HIV infection, and he was referred to the Division of Infectious Diseases of the Musashino Red Cross Hospital in Tokyo, Japan. The patient's medical history included aortic and mitral valve replacement as a result of aortic and mitral stenosis. On physical examination, his vital signs were normal, and he had bilateral, painless parotid gland swelling (figure 1). The right parotid gland measured approximately $4 \times 3 \mathrm{~cm}$ and the left parotid gland measured approximately $4 \times 2.5 \mathrm{~cm}$. Laboratory tests conducted at the initial presentation included western blotting, CD4 T lymphocyte (CD4) cell count and HIV viral load, and the results were as follows: HIV-1 (+); CD4 237 cells/ $\mu \mathrm{L}$; and HIV-1 RNA 30,000 copies/mL, respectively. Contrast-enhanced CT showed multiple cysts in the bilateral parotid glands (figure 2). A biopsy of the parotid gland was performed to confirm diagnosis. Histologically, the lesion consisted of a cyst wall lined with stratified epithelium and accompanied by dense lymphoid tissue. The lymphoid tissue contained lymphoid follicles with conspicuous germinal centres (figure 3). Immunohistochemical studies revealed that the dendritic cells in the germinal centres were positive for HIV-1 p24 antigen, suggesting that they were HIV-infected (figure 4). This antigen was made by the Department of Pathology, National Institute of Infectious Diseases. ${ }^{3}$ Immunohistochemical studies for the human herpesvirus- 8 latency-associated nuclear antigen were negative. Furthermore, in situ hybridization for Epstein-Barr virus-encoded small RNA was negative. The patient was subsequently diagnosed with HIV-associated BLEC.

\section{OUTCOME AND FOLLOW-UP}

He was started on ART (tenofovir disoproxil fumarate, emtricitabine and dolutegravir), 


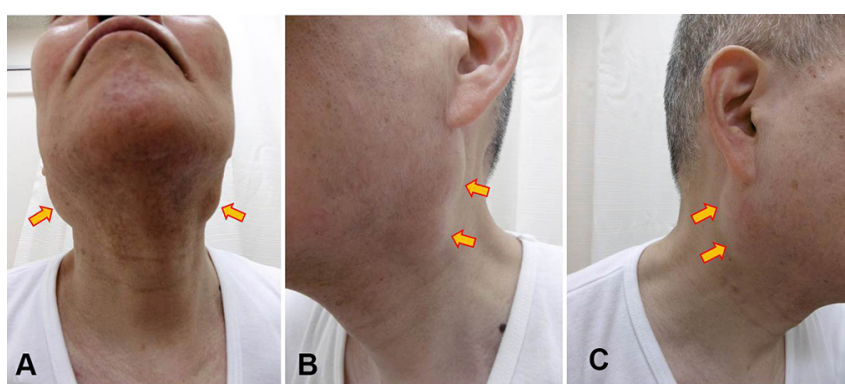

Figure 1 Bilateral parotid glands with swelling (indicated by the arrows; frontal (A), left (B) and right (C) view).

and the parotid glands dramatically decreased in size 6 months after administration of ART (figure 5).

\section{DISCUSSION}

BLEC is a cystic disease of the parotid glands that can be differentially diagnosed as Warthin's tumours, and cystic degeneration of benign and malignant tumours. HIV-associated BLEC is believed to be a local manifestation of persistent generalised lymphadenopathy associated with HIV infection. Although BLEC is a rare manifestation of HIV, it can serve as the first clinical manifestation of the virus.

While the proliferation of BLEC has been shown to increase with HIV replication, the exact pathology of this disease remains unknown. ${ }^{4}$ Currently, there are two hypotheses about the pathology of BLEC. The first hypothesis is that BLECs are solitary, or multiple cysts within lymph nodes trapped during the parotid gland embryogenesis. HIV-related reactive lymphoproliferation occurs in the intraparotid lymph nodes of the parotid glands. The parotid glandular epithelium becomes trapped within the normal intraparotid lymph nodes, resulting in cystic enlargement. ${ }^{56}$ The second hypothesis is that HIV-infected cells migrate into the parotid glands. This migration triggers lymphoid proliferation and salivary duct dysplasia, which induces ductal obstruction. Cysts are subsequently formed due to ductal obstruction. ${ }^{7}$ These cysts serve as a reservoir of HIV-1 p24 antigen and RNA copies that are about 1000-fold higher than plasma concentrations. ${ }^{6}$ HIV has a predilection for lymphoid tissue and elevated concentrations of HIV can be found within these nodes. ${ }^{8}$

BLEC can be defined as the occurrence of single or multiple cysts in the parotid gland lymph nodes. The clinical presentation of BLEC is typically gradual, painless and comprises bilateral parotid swelling with diffuse cervical lymphadenopathy.
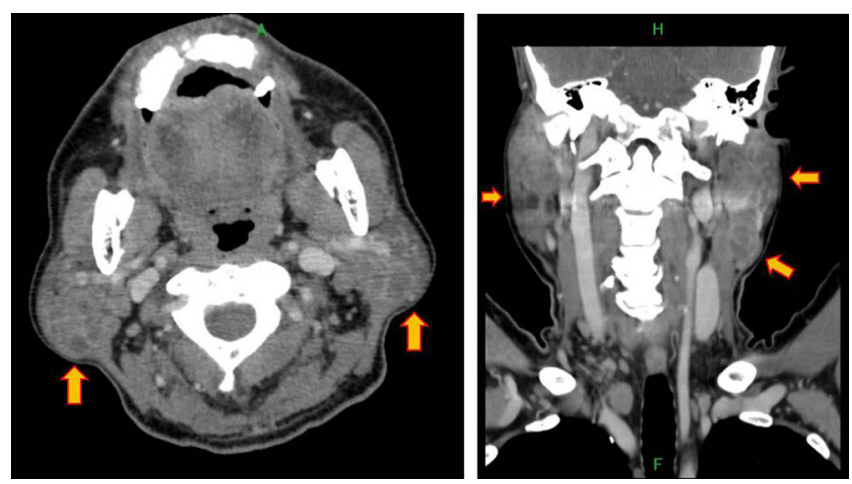

Figure 2 Parotid glands with multiple cysts (indicated by the arrows) on contrast-enhanced CT.

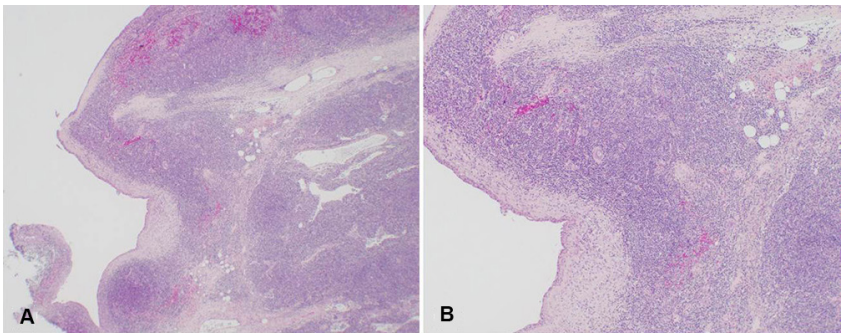

Figure 3 H\&E staining revealed the parotid gland cyst had an undulating stratified squamous epithelium surrounded by dense lymphoid tissue (original magnification was $\times 20(A)$ and $\times 40(B)$ ).

Unilateral parotid swelling has also been reported ${ }^{9}$; however, it is usually found in HIV-negative patients, and rarely found in HIV-positive patients. Most patients who develop BLEC are generally not aware of potential HIV infection, but are more concerned with the cosmetic implications. However, BLEC rarely causes facial palsy and xerostomia, and if patients present with systematic symptoms, such as fever, weight loss or night sweats, they should be examined for tuberculosis or lymphoma.

BLEC is diagnosed based on medical history, physical examination and biopsy. Non-invasive diagnostic modalities include ultrasound examination, CT scanning and MRI, which are able to detect multiple thin-walled cysts with diffuse cervical lymphadenopathy. Invasive diagnostic modalities include fine needle aspiration cytology (FNAC) and parotid gland biopsy, which can exclude malignancy in the differential diagnosis. However, malignancy only occurs in less than $1 \%$ of patients with HIV-associated cystic lesions. ${ }^{10}$ FNAC of BLEC is a safe examination that usually reveals a heterogeneous cell population, which consists of scattered foamy macrophages and anucleated squamous cells in a proteinaceous background. ${ }^{11}$ When a small amount of sample is obtained, it may be difficult to exclude malignant diseases such as lymphoma, Warthin's tumour and cystic metastatic squamous cell carcinoma by FNAC. Since biopsy often includes the collection of multiple tissue specimens, it may have a higher sensitivity to diagnosis than FNAC. Thus, pathological tissue examination is the only method that definitively diagnose BLEC.

Histologically, lymphoepithelial cysts typically consist of multiple epithelial cysts accompanied by dense lymphoid tissue. The epithelial lining can be squamous, cuboidal or columnar, and although some cysts have pseudostratified ciliated epithelium, stratified squamous epithelium is the most common. The lymphoid tissue replaces normal parotid parenchyma, and the lymphoid follicles are larger and more irregularly shaped than unreactive follicles in normal lymph nodes. The germinal centres often contain numerous macrophages, and some of the germinal centres have an attenuated mantle of small

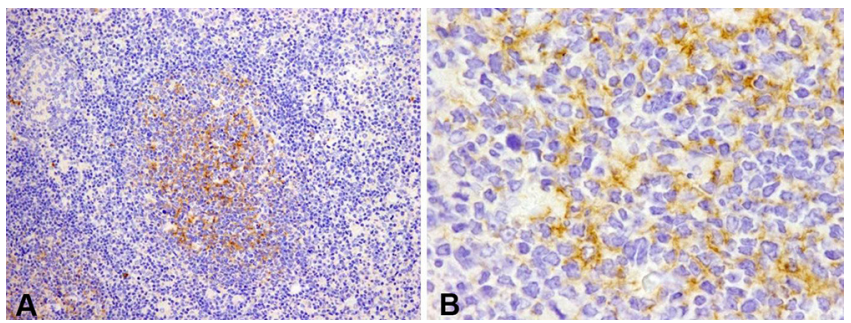

Figure 4 Immunohistochemical test results revealed HIV-1 p24 antigen in the follicular centre, and the dendritic cells in the follicular centres were positive for HIV-1 p24 antigen (original magnification was $\times 100(A)$ and $\times 400(B))$. 

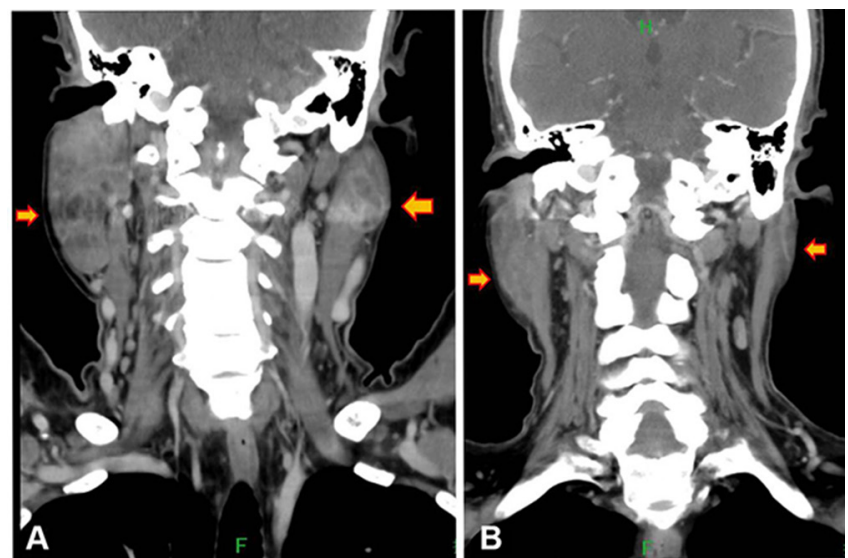

Figure 5 The size of the BLEC decreased 6 months after starting ART (before ART (A) and 6 months after ART (B)). (B) The size and number of BLEC decreased, and the size of the parotid glands (indicated by the arrows) also decreased. ART, antiretroviral therapy; BLEC, benign lymphoepithelial cyst.

lymphocytes that invaginates into the follicles. ${ }^{12} 13$ While the number of lymphoepithelial cysts varies, they are not pathognomonic of HIV-associated lymphoepithelial cysts and similar features occur in some cases of HIV-negative lymphoepithelial sialadenitis. HIV-1 p24 antigen immunostaining is a useful additional study as the follicular dendritic cells and interfollicular macrophages in most lesions are positive for HIV-1 p24 antigen. $^{13} 14$

Since most patients with BLEC are asymptomatic, treatment intervention is often indicated by cosmetic issues. However, the management of BLEC remains controversial. BLEC treatment options include repeat aspiration, ART, sclerotherapy, radiation therapy and surgery. Repeat aspiration can be easily and quickly performed in a clinical setting, but most of aspirated cysts will grow back after several weeks or months. In contrast, ART can reduce or eliminate the cysts entirely. ${ }^{15} \mathrm{HIV}$ is assumed to replicate more in parotid gland lymphatic tissues because the HIV-1 p24 antigen is detected in the dendritic cells, meaning the size of the BLEC can be reduced by ART. Sclerotherapy is a treatment option wherein the cysts are injected with multiple sclerotic agents, such as sodium morrhuate, doxycycline, ethanol, bleomycin and picibanil. ${ }^{7}$ Several studies have shown that sclerotherapy with doxycycline or bleomycin can reduce cyst size by $40 \%-100 \%$ without serious complications. ${ }^{2}$ Radiation therapy is another therapeutic modality. Beitler et $a l^{16}$ reported that low-dose external radiotherapy of 18-24 Gy resulted in partial cosmetic improvement in 14 out of 20 patients with side effects of xerostomia and mucositis. Still, some patients choose surgery because BLEC recurrence is possible with the previously described treatments. Steehler et $a l^{7}$ reported parotidectomy to be the gold standard treatment because it was the only method to show a complete response with no recurrence. However, some reports do not recommend surgery as a first-line treatment because there is a $2 \%-7 \%$ risk of facial nerve injury, bleeding and seroma formation. Therefore, surgery may be a good option in cases of possible malignancy or of poor patient response to the treatments listed above. ${ }^{28}$

In the present case, the parotid gland cyst walls had an undulating and irregular surface, and immunohistochemical detection revealed that the germinal centre dendritic cells were positive for HIV-1 p24 antigen. Furthermore, biopsy did not indicate malignant tumour. The size of the parotid tumour dramatically decreased after starting ART, and his cosmetic issues were resolved.

\section{Learning points}

- Patients with painless, bilateral parotid swelling should be considered for HIV infection.

- Bilateral parotid swelling in patients with HIV is usually clinically diagnosed as benign lymphoepithelial cyst (BLEC). However, HIV-associated BLEC is best confirmed via biopsy because other salivary gland diseases, such as malignant lymphoma, can be present. Immunohistochemical detection of HIV-1 p24 antigen definitively diagnose HIV-associated BLEC.

- We report a rare case of HIV-associated BLEC confirmed on the basis of immunohistochemical detection of HIV-1 p24 antigen. Thus, immunohistochemical detection for HIV-1 p24 should be considered in patients with HIV presenting with bilateral parotid swelling to confirm HIV-associated BLEC.

Acknowledgements The authors wish to thank Dr. Urara Sakurai of the Department of Pathology at Musashino Red Cross Hospital in Tokyo, Japan, and Dr. Harutaka Katano of the Department of Pathology at the National Institute of Infectious Diseases in Tokyo, Japan, for help interpreting this patient's pathology findings.

Contributors YS wrote the initial draft of the manuscript. IH contributed to analysis and interpretation of data, and assisted in the preparation of the manuscript. Both authors approved the final version of the manuscript.

Competing interests None declared.

\section{Patient consent Obtained.}

Provenance and peer review Not commissioned; externally peer reviewed.

Open Access This is an Open Access article distributed in accordance with the Creative Commons Attribution Non Commercial (CC BY-NC 4.0) license, which permits others to distribute, remix, adapt, build upon this work non-commercially, and license their derivative works on different terms, provided the original work is properly cited and the use is non-commercial. See: http://creativecommons.org/ licenses/by-nc/4.0/

(C) BMJ Publishing Group Ltd (unless otherwise stated in the text of the article) 2017. All rights reserved. No commercial use is permitted unless otherwise expressly granted.

\section{REFERENCES}

1 Frezzini C, Leao JC, Porter S. Current trends of HIV disease of the mouth. J Oral Pathol Med 2005;34:513-31.

2 Shanti RM, Aziz SR. HIV-associated salivary gland disease. Oral Maxillofac Surg Clin North Am 2009;21:339-43.

3 Nakajima N, lonescu P, Sato Y, et al. In situ hybridization AT-tailing with catalyzed signal amplification for sensitive and specific in situ detection of human immunodeficiency virus-1 mRNA in formalin-fixed and paraffin-embedded tissues. Am J Pathol 2003:162:381-9.

4 Mandel L, Reich R. HIV parotid gland lymphoepithelial cysts. Review and case reports. Oral Surg Oral Med Oral Pathol 1992:74:273-8.

5 Bernier JL, Bhaskar SN. Lymphoepithelial lesions of salivary glands; histogenesis and classification based on 186 cases. Cancer 1958;11:1156-79.

6 Shivhare P, Shankarnarayan L, Jambunath U, et al. Benign lymphoepithelial cysts of parotid and submandibular glands in a HIV-positive patient. J Oral Maxillofac Pathol 2015;19:107.

7 Steehler MK, Steehler MW, Davison SP. Benign lymphoepithelial cysts of the parotid: long-term surgical results. Hiv Aids 2012;4:81-6.

8 Ebrahim S, Singh B, Ramklass SS. HIV-associated salivary gland enlargement: a clinical review. SADJ 2014:69:400-3.

9 Dave SP, Pernas FG, Roy S. The benign lymphoepithelial cyst and a classification system for lymphocytic parotid gland enlargement in the pediatric HIV population. Laryngoscope 2007;117:106-13

10 Madani G, Beale T. Inflammatory conditions of the salivary glands. Semin Ultrasound CT MR 2006;27:440-51

11 Michelow P, Dezube BJ, Pantanowitz L. Fine needle aspiration of salivary gland masses in HIV-infected patients. Diagn Cytopathol 2012;40:684-90. 
12 Chetty R. HIV-associated lymphoepithelial cysts and lesions: morphological and immunohistochemical study of the lymphoid cells. Histopathology 1998;33:222-9.

13 Chetty R, Vaithilingum M, Thejpal R. Epstein-Barr virus status and the histopathological changes of parotid gland lymphoid infiltrates in HIV-positive children. Pathology 1999;31:413-7.

14 Labouyrie E, Merlio JP, Beylot-Barry M, et al. Human immunodeficiency virus type 1 replication within cystic lymphoepithelial lesion of the salivary gland. Am I Clin Pathol 1993;100:41-6.
15 Syebele K, Bütow KW. Comparative study of the effect of antiretroviral therapy on benign lymphoepithelial cyst of parotid glands and ranulas in HIV-positive patients. Oral Surg Oral Med Oral Pathol Oral Radiol Endod 2011:111:205-10.

16 Beitler JJ, Vikram B, Silver CE, et al. Low-dose radiotherapy for multicystic benign lymphoepithelial lesions of the parotid gland in HIV-positive patients: long-term results. Head Neck 1995;17:31-5.

Copyright 2017 BMJ Publishing Group. All rights reserved. For permission to reuse any of this content visit http://group.bmj.com/group/rights-licensing/permissions.

BMJ Case Report Fellows may re-use this article for personal use and teaching without any further permission.

Become a Fellow of BMJ Case Reports today and you can:

- Submit as many cases as you like

- Enjoy fast sympathetic peer review and rapid publication of accepted articles

Access all the published articles

- Re-use any of the published material for personal use and teaching without further permission

For information on Institutional Fellowships contact consortiasales@bmjgroup.com

Visit casereports.bmj.com for more articles like this and to become a Fellow 